\title{
Recurrent Urinary Tract Infection in a Patient With Asymptomatic Crohn's Disease
}

\author{
Waqar Akram ${ }^{1}$, Sanket K. Shah ${ }^{1}$, Mohina Sohail ${ }^{1}$, Usama Rehman ${ }^{2}$, Mustafa Rahim ${ }^{3}$ \\ 1. Internal Medicine, Raleigh General Hospital, Beckley, USA 2. Anesthesia, Mayo Hospital, Lahore, PAK 3. Internal \\ Medicine, West Virginia University School of Medicine, Morgantown, USA
}

Corresponding author: Waqar Akram,waqarshoaib@hotmail.com

\begin{abstract}
Recurrent urinary tract infection (UTI) is uncommon in males, but it is a common complication of Crohn's disease (CD). Patients with CD often present with abdominal pain, diarrhea, and systemic symptoms, such as weight loss, low-grade fever, and fatigue, and rarely it can cause serious complications, such as fistulas or abscesses. Some patients with CD remain asymptomatic, which can progress to severe complications and delayed treatment. We are presenting a 22 -year-old male with recurrent UTIs and no established past medical history of $\mathrm{CD}$. However, on subsequent investigations, an anatomical abnormality was discovered that helped us make the diagnosis of CD. The aim of this report is to emphasize the early detection of asymptomatic $\mathrm{CD}$ in atypical patients, to not only decrease the risk of complications such as UTIs but also allow for early treatment intervention and better outcomes.
\end{abstract}

Categories: Internal Medicine, Medical Education, Gastroenterology

Keywords: crohn's disease, urinary tract infection

\section{Introduction}

Urinary tract infection (UTI) is uncommon in male patients younger than 50 years. The causes of UTI include prostatitis, epididymitis, orchitis, pyelonephritis, cystitis, arthritis, and urinary catheters. Many experts believe that UTI in males is associated with anatomic abnormalities and might require surgical procedures to avoid further complications. Obtaining a urine culture in a suspected male UTI can help narrow down the diagnosis. In a majority of males with their first UTI, imaging adds little to supplement a careful history and physical exam. Men below 45 years of age with their first UTI who respond well to antibiotic treatment are not likely to have a urologic abnormality $[1,2]$.

Received 08/14/2020

Review began 08/19/2020 Review ended 08/19/2020 Published 08/23/2020

๑) Copyright 2020

Akram et al. This is an open access article distributed under the terms of the Creative Commons Attribution License CC-BY 4.0., which permits unrestricted use, distribution, and reproduction in any medium, provided the original author and source are credited.
An estimated $1.3 \%$ of adults in the United States are diagnosed with inflammatory bowel disease (IBD) either with CD or ulcerative colitis (UC). The risk of CD slightly increases in women and an Ashkenazi Jewish compared to non-Jews [2]. Age above 45 years, living below poverty, sedentary lifestyle, and unemployment are some of the commonly reported risk factors. Patients with CD often present with abdominal pain and diarrhea and may develop systemic symptoms, such as weight loss, low-grade fever, and fatigue. Fistulas or abscesses can develop in a patient with CD. Patient symptoms associated with the location of fistulae such as enterovesicular or enterourethral fistula present with recurrent UTI, enterovaginal fistula form passage of stool, enterocutaneous fistula cause drainage through the skin, and enteroenteric fistula cause diarrhea which can be bloody in severe cases [2,3]. Our aim in this report is to stress the importance of taking into consideration asymptomatic CD and pursing early detection in atypical patients presenting with recurrent UTIs. This will allow for early treatment intervention that will lead to decreased risk of complications and improved outcomes.

\section{Case Presentation}

A 22-year-old male patient who came to the hospital with recurrent UTI and hematuria complains of nausea, vomiting, flank pain, increased frequency of urination, cloudy urine, dysuria, and intermittent blood in the stool. However, he denied fatigue, fever or chills, abdominal pain, weight loss, constipation, diarrhea, urethral discharge, and pain radiating to the groin. The patient had no known medical or family history. The patient is a non-smoker, is sexually active with intermittent protection usage, and drinks alcohol occasionally.

On initial assessment, it appeared to be pyelonephritis and sexually transmitted infection (STI). However, STI was ruled out by history and lab tests. Imaging using CT and CT scan with contrast showed fistulas and the presence of an enterovesicular fistula. Axial CT scan revealed thickened pseudodiverticulum wall suggesting fistula (Figure 1). 


\section{Cureus}

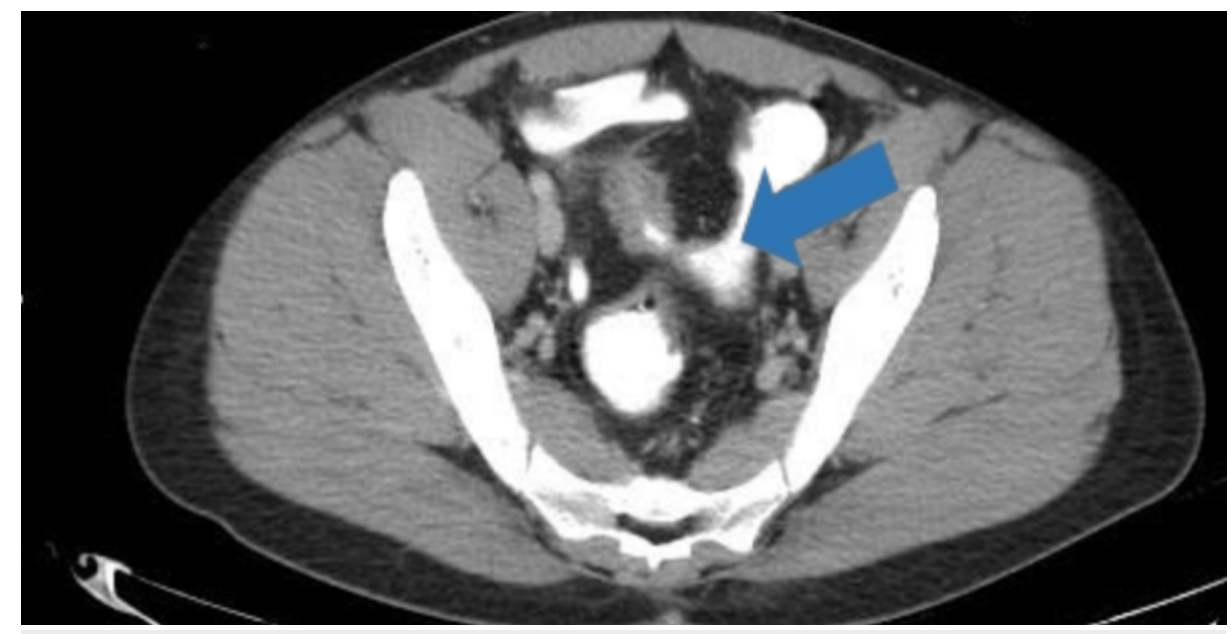

FIGURE 1: CT scan showing distal ileum with pseudodiverticulum thickened wall indicated by the blue arrow

Coronal scan of the abdomen shows thickened sigmoid colon suggesting chronic inflammation by bowel disease (Figure 2).

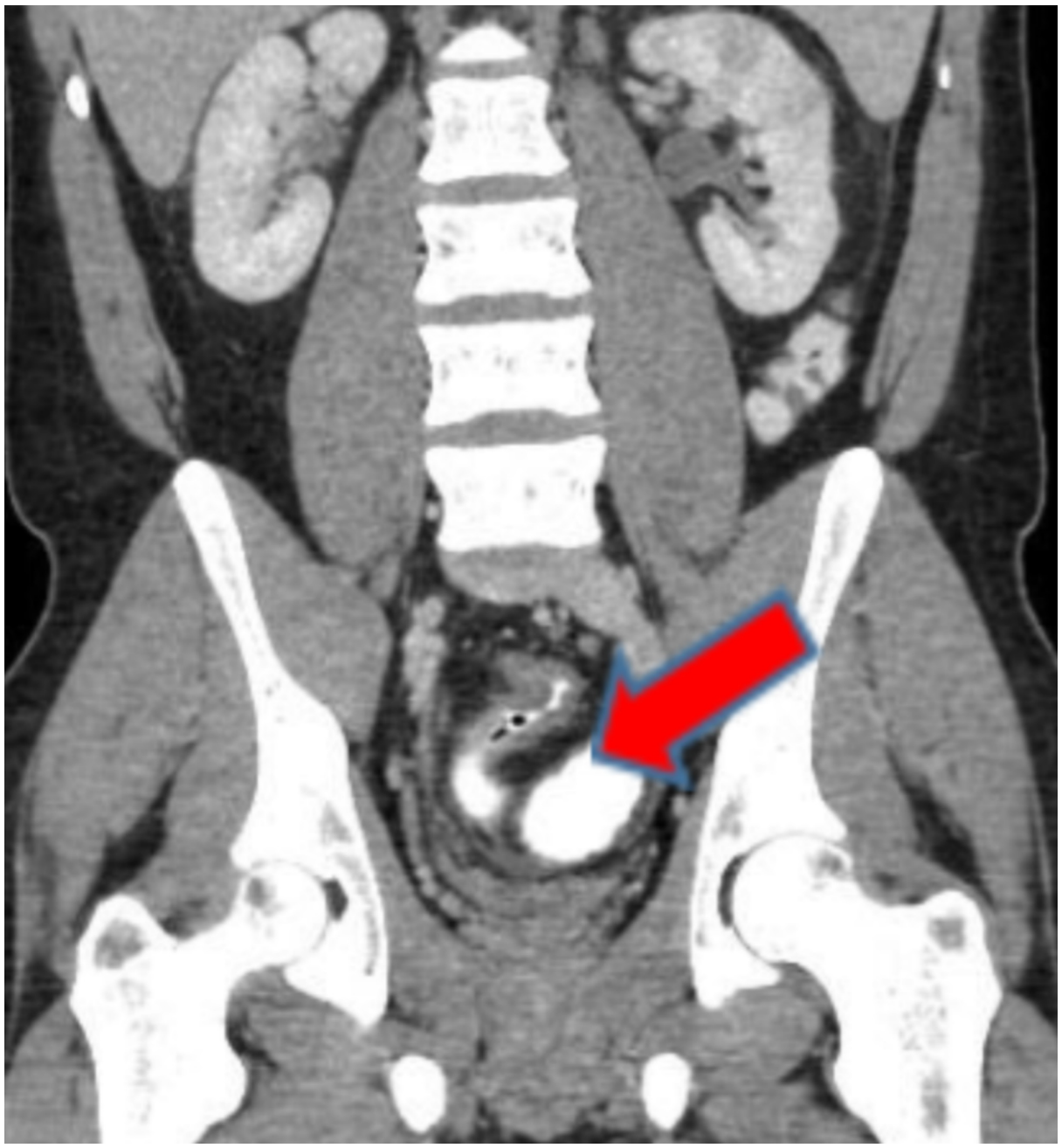

FIGURE 2: CT scan showing abnormal wall thickening of sigmoid colon indicated by the red arrow 


\section{Cureus}

Fistulas between small and large bowel suggest colonic fistula on CT scan (Figures 3, 4).

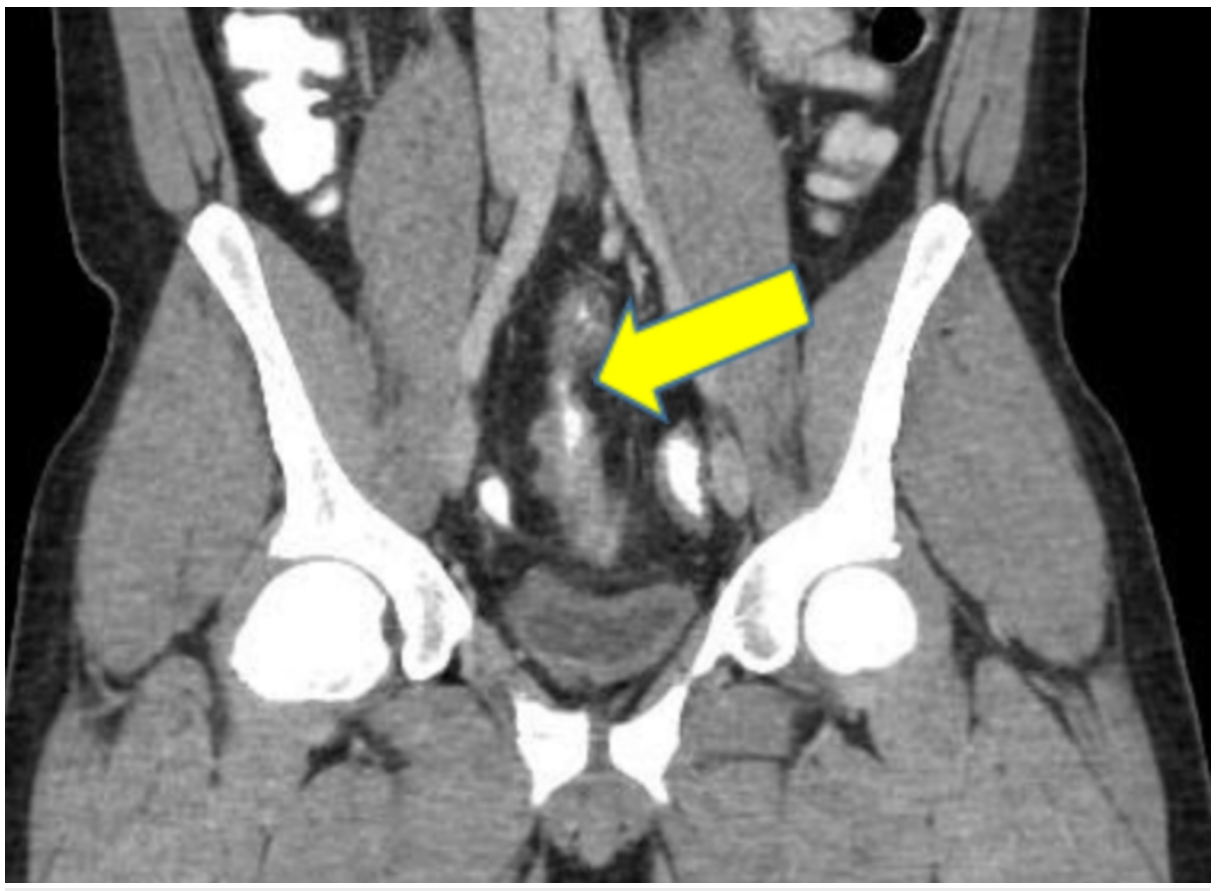

FIGURE 3: CT scan showing fistula between terminal ileum and sigmoid colon indicated by the yellow arrow

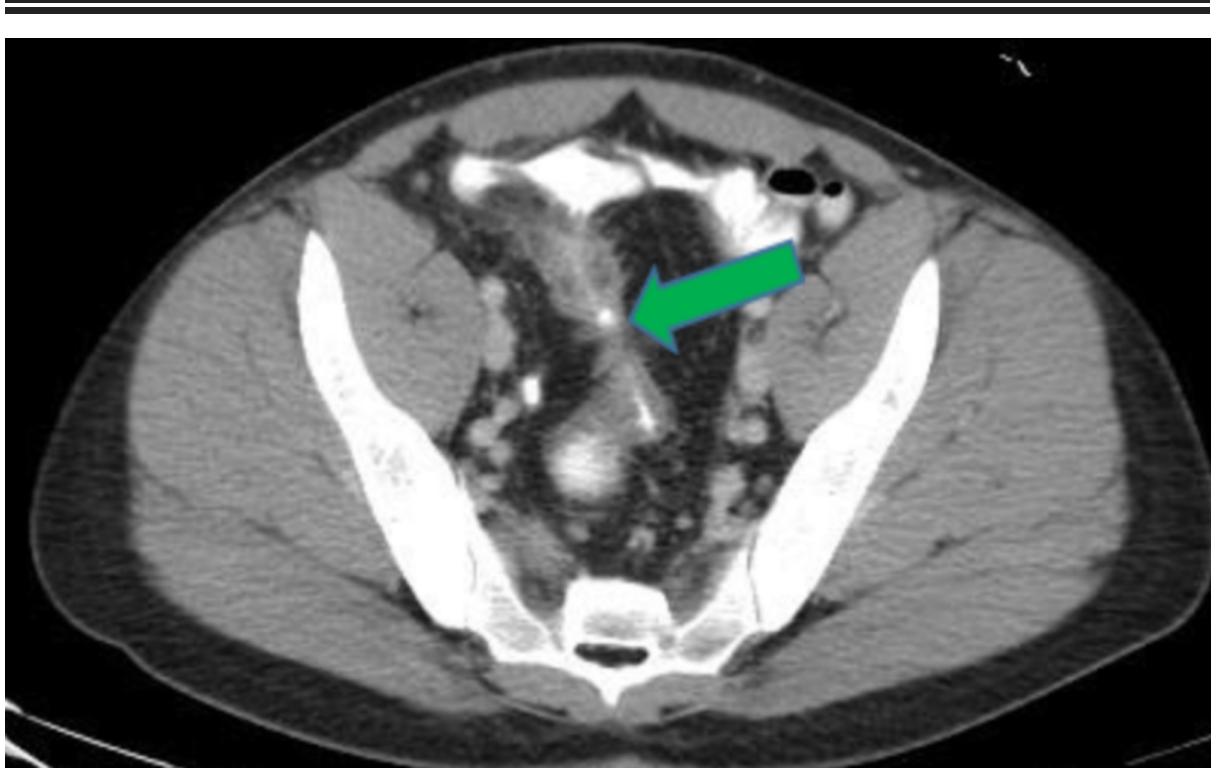

FIGURE 4: CT scan showing fistula connecting bowels indicated by the green arrow

Axial CT scan also showed the presence of a fistula between the bladder and the terminal ileum suggesting enterovesicular fistula (Figure 5). 


\section{Cureus}

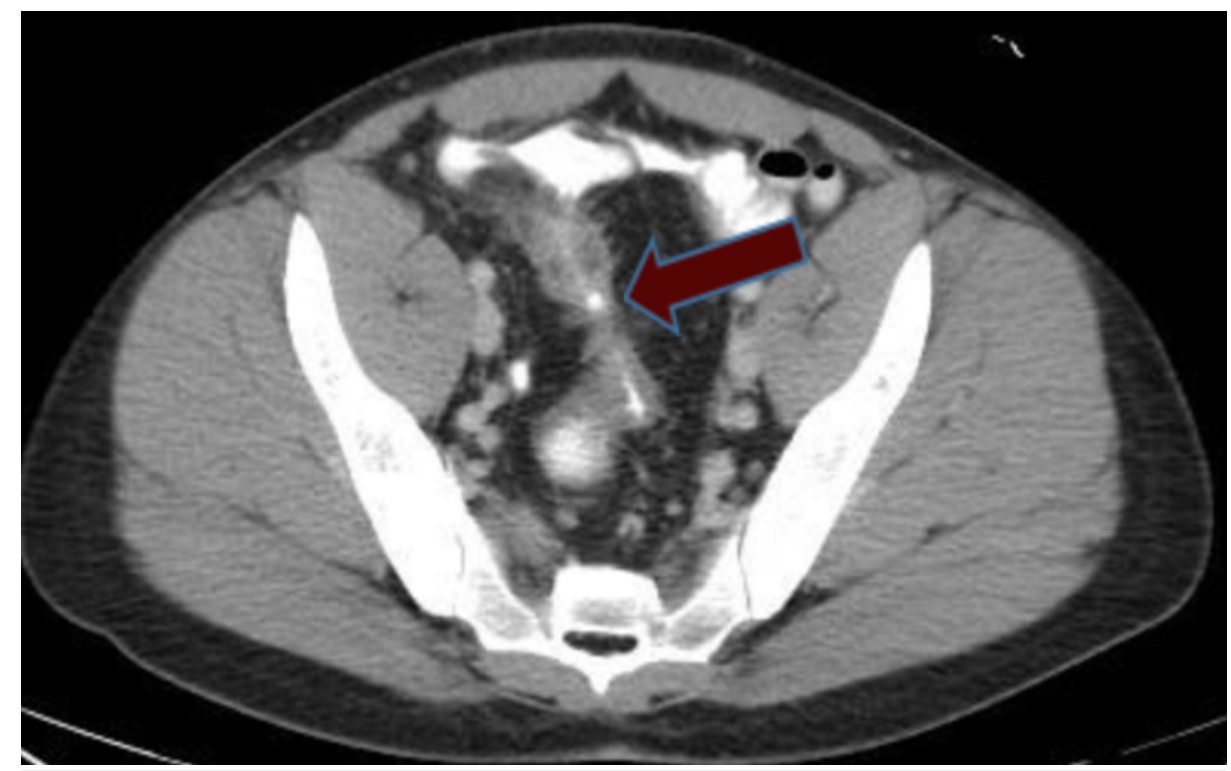

FIGURE 5: CT scan showing presence of fistula between the bladder and terminal lleum indicated by the red arrow

Sagittal CT scan shows the presence of thickened sigmoid colon suggesting chronic inflammation (Figure 6).

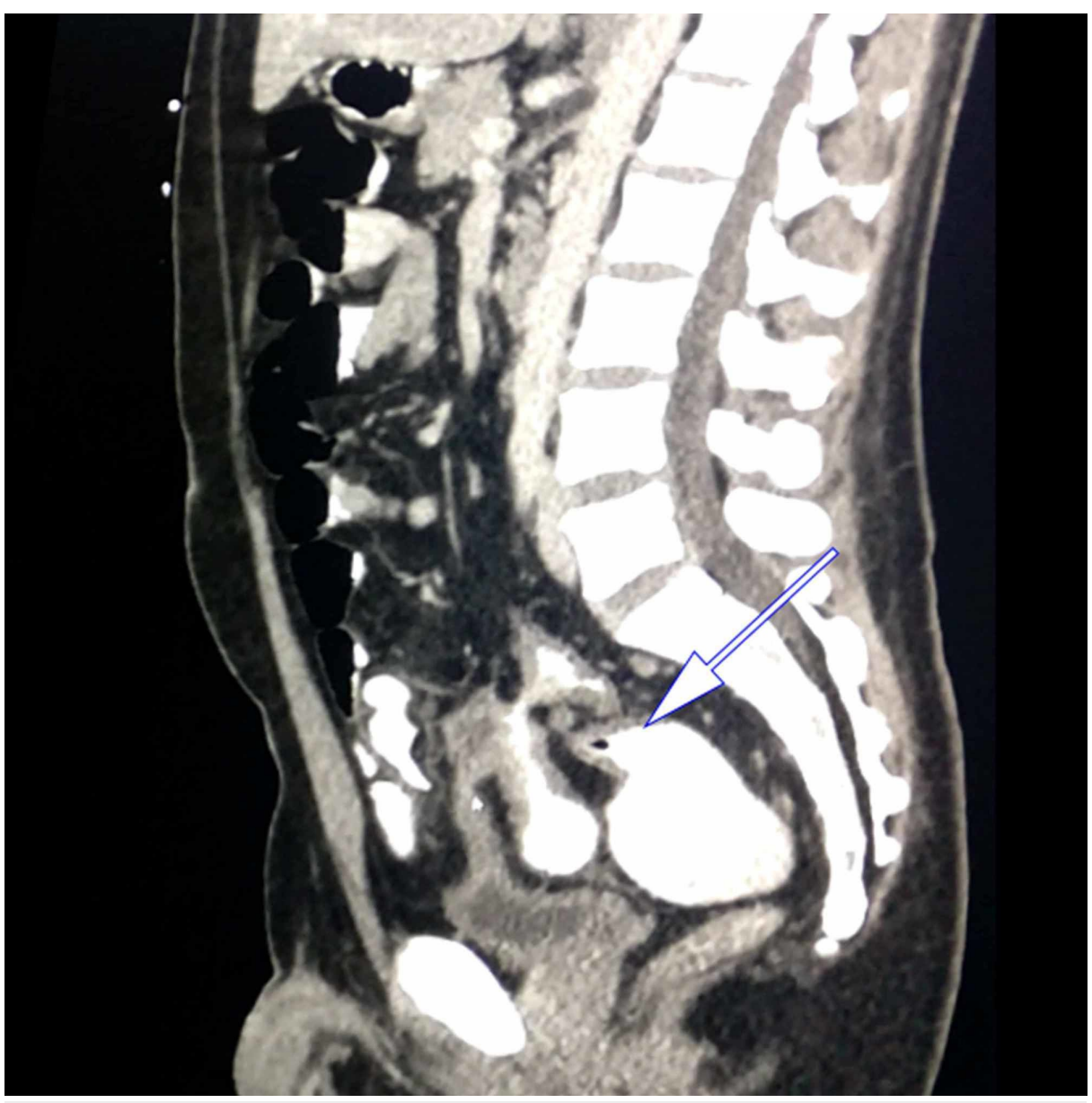

FIGURE 6: CT scan showing thickened sigmoid colon indicated by the white arrow 


\section{Cureus}

The presence of hypodensity on a bilateral kidney CT scan is suggestive of pyelonephritis. Based on the CT findings and the presence of fistulas, the patient was diagnosed with $\mathrm{CD}$ (Figure 7).

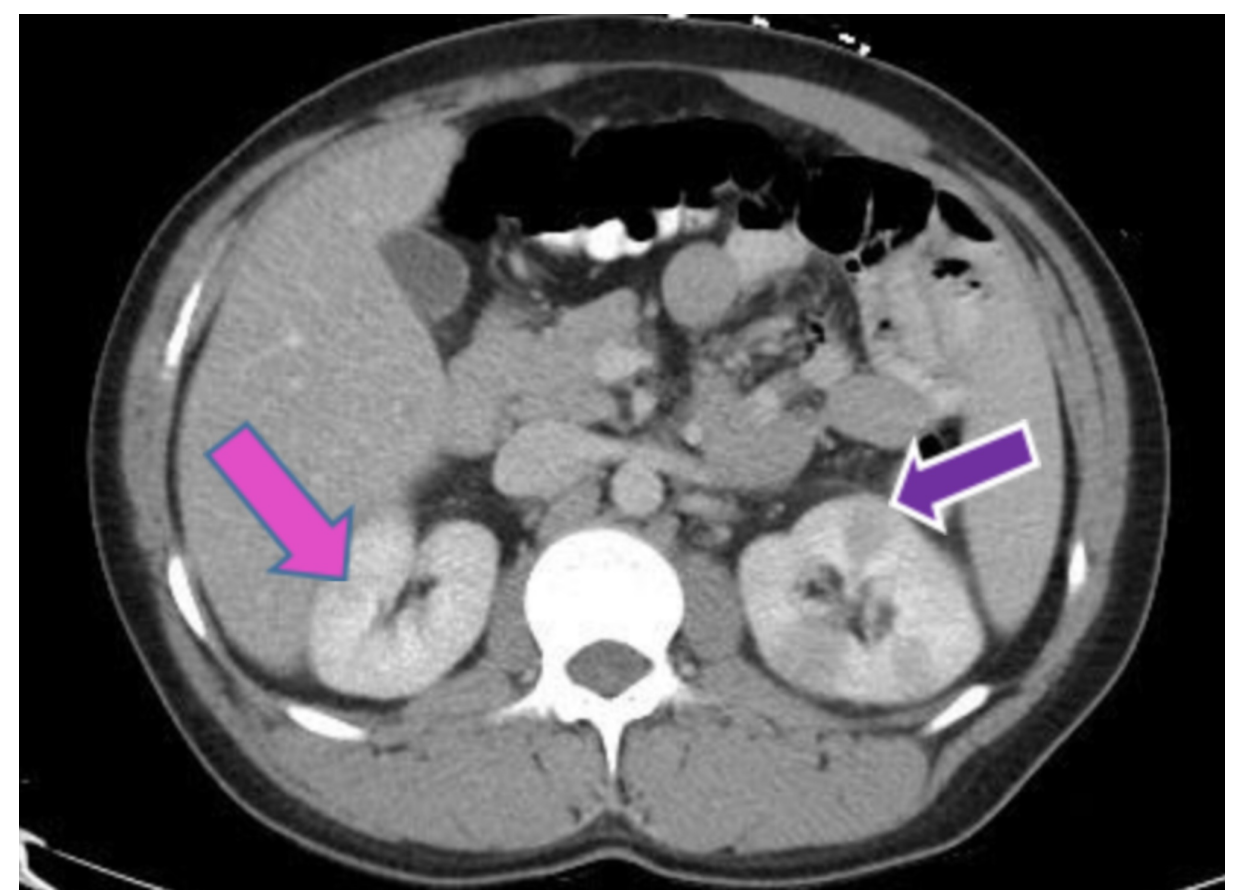

FIGURE 7: CT scan showing darkening of kidneys shows pyelonephritis indicated by the pink and purple arrows

\section{Discussion}

An incidence of UTI in males is five to eight cases per year per 10,000 cases with ages between 21 and 50 years, and prevalence is about $14 \%$. Patients diagnosed with first UTI, with high-risk factors such as immunocompromised status, uncircumcised, engaging in anal intercourse, greater than 65 years of age, bladder outlet obstruction, anatomical functional abnormalities, previous urinary tract surgery, cystoscopy, catheterization, or transrectal prostate biopsy, may not need further unnecessary evaluation. Limiting further evaluation of men with a first UTI to those at increased risk may reduce unnecessary radiological, endoscopic, or urodynamic investigation. There is minimal evidence to support routine imaging in low-risk men with a first UTI, whether with or without fever [1,2].

Recurrent UTI in men warrants evaluation for structural abnormalities, colovesical fistulas associated with colonic malignancy or IBD, enlarged prostate, and unprotected sexual intercourse. Screening for immunologic disorders that might increase the risk of infection with the human immunodeficiency virus and hematologic malignancies is also recommended [3].

Organisms causing UTI can suggest the etiology. Gram-negatives such as Escherichia coli, Klebsiella pneumoniae, Pseudomonas aeruginosa, and Proteus mirabilis are often found in anatomical obstruction. On the other hand, organisms such as Chlamydia trachomatis, Neisseria gonorrhoeae, Trichomonas vaginalis, or Ureaplasma urealyticum are typically STIs. Yeast infections suggest underlying immunosuppression that occurs with diabetes or corticosteroid use [3].

CD follows a bimodal age distribution, with the first peak between the ages 15 and 30 years. Clinically, $C D$ presents with abdominal pain, reduced appetite, diarrhea with blood in the stool, and weight loss. In patients who present with recurrent UTIs and are asymptomatic, physicians should always keep CD in mind, imaging techniques like CT scan and MRI for the extent of fistulas should be used and they decrease the high morbidity associated with it $[1,4]$.

The European Crohn's and Colitis Organization (ECCO) and the European Society of Gastrointestinal and Abdominal Radiology (ESGAR) published new imaging guidelines for $\mathrm{CD}$. The diagnosis of $\mathrm{CD}$ and $\mathrm{UC}$ requires multiple steps, which include clinical evaluation, stool sampling, endoscopy, imaging study, and histological findings. Multiple imaging studies help to establish diagnoses such as MRI, CT scan, magnetic resonance enterography (MRE), endoscopy, and colonoscopy. MRI is the first line study used to diagnose and classify perianal $\mathrm{CD}$ and provide a more accurate assessment of abscess and pelvic fistula. Transrectal ultrasounds are better than clinical exam in providing better information. A combination of MRI and 
Abdominal ultrasound, MRE, or a small bowel capsule endoscopy (SBCE) helps evaluate to newly diagnose a patient with $\mathrm{CD}$ and can be used in patients with negative endoscopy. Biopsies of both inflamed and noninflamed segments are required to establish an accurate diagnosis. Patients with high clinical suspicion of $\mathrm{CD}$ and normal biopsy should be considered for SBCE or cross-sectional imaging. The presence of at least three small bowel ulcers on SBCE in a patient who has not used non-steroidal anti-inflammatory drugs for one month has a high chance of having CD $[1,6]$.

The diagnostic studies, such as CT enterography and MRE, provide information that helps to treat a patient based on the severity and extent of $\mathrm{CD}$ and helps to evaluate its complications that cannot be observed by clinical exam or endoscopic evaluation of kids and adults. Based on the severity of the presentation, CD requires medical management or surgical intervention. For enterovesical fistula due to $\mathrm{CD}$, medical therapy is the first choice. Zhang et al. reported long-term remission of enterovesical fistula in 13 of 37 patients with CD over a mean of 4.7 years through treatment with antibiotics, azathioprine, steroids, and infliximab [3,7-10].

Antibiotic therapy should be based on the organism and sensitivities when available. Fluoroquinolone and trimethoprim-sulfamethoxazole are first-line agents. Ustekinumab is a monoclonal antibody to the p40 subunit of interleukin-12 and interleukin-23 used in a patient with moderate to severely active CD. Research by Feagan et al. found that ustekinumab provides systemic anti-inflammatory activity, better safety profile, and has a lack of immunogenicity [11]. It is administered as one single dose intravenously, and subsequent use of subcutaneous injections provides a home-based advantage. It is more beneficial for a patient with the extraintestinal manifestation of disease and decreases the risk of infection. Ustekinumab had a higher response rate compared to placebo and used as maintenance therapy [3,5,8-11].

Definite treatment of enterovesical fistula is the surgical resection of the involved bowel. Gaglani et al. concluded that laparoscopic resection of IBD decreases hospital stays and operating time along with decreased complications [7]. It can offer a safer treatment possibility for IBD-associated fibrotic stricture. There is no need to repair the bladder wall because the vesical portion heals spontaneously. A patient who denies surgical repair can be managed by medical therapy $[3,11]$.

\section{Conclusions}

The initial workup for UTI consists of urine analysis and culture, but does not include imaging as it does not add diagnostic value. However, in atypical patients presenting with recurrent UTIs, after ruling out common etiologies, we recommend clinicians consider asymptomatic CD leading to an enterovesicular fistula. Extensive investigation using imaging techniques like X-ray, CT scan, MRI, MRE, and SBCE can be useful in establishing a diagnosis of CD. Pursuing early detection of asymptomatic CD in such atypical patients will allow for early intervention that can help reduce severe complications and improve outcomes.

\section{Additional Information \\ Disclosures}

Human subjects: Consent was obtained by all participants in this study. Conflicts of interest: In compliance with the ICMJE uniform disclosure form, all authors declare the following: Payment/services info: All authors have declared that no financial support was received from any organization for the submitted work. Financial relationships: All authors have declared that they have no financial relationships at present or within the previous three years with any organizations that might have an interest in the submitted work. Other relationships: All authors have declared that there are no other relationships or activities that could appear to have influenced the submitted work.

\section{Acknowledgements}

We are thankful to Dr. Muhammad Uzair Lodhi MD and Dr. Hasnan Ijaz MD for their critical review of this report.

\section{References}

1. Breen DP, Wanserski GR, Smith PC: Clinical inquiries. What is the recommended workup for a man with a first UTI?. J Fam Pract. 2007, 56:657-661.

2. Feuerstein JD, Cheifetz AS: Crohn disease: epidemiology, diagnosis, and management. Mayo Clin Proc. 2017, 92:1088-1103. 10.1016/j.mayocp.2017.04.010

3. Seminerio JL, Aggarwal G, Sweetser S: 26-year-old man with recurrent urinary tract infections . Mayo Clin Proc. 2011, 86:557-560. 10.4065/mcp.2010.0600

4. Schäffler H, Herlemann DP, Klinitzke P, Berlin P, Kreikemeyer B, Jaster R, Lamprecht G: Vitamin D administration leads to a shift of the intestinal bacterial composition in Crohn's disease patients, but not in healthy controls. J Dig Dis. 2018, 19:225-234. 10.1111/1751-2980.12591

5. Testing and diagnosis - imaging techniques . (2019). Accessed: February 2, 2020: 


\section{Cureus}

https://crohnsandcolitis.ca/About-Crohn-s-Colitis/Testing-diagnosis

6. Rubin DT, Ananthakrishnan AN, Siegel CA, Sauer BG, Long MD: ACG clinical guideline ulcerative colitis in adults. Am J Gastroenterol. 2019, 114:384-413. 10.14309/ajg.0000000000000152

7. Gaglani T, Davis CH, Bailey HR, Cusick MV: Trends and outcomes for minimally invasive surgery for inflammatory bowel disease. J Surg Res. 2019, 235:303-307. 10.1016/j.jss.2018.09.075

8. Bruining DH, Zimmermann EM, Loftus EV, Sandborn WJ, Sauer CG, Strong SA, Society of Abdominal Radiology Crohn's Disease-Focused Panel: Consensus recommendations for evaluation, interpretation, and utilization of computed tomography and magnetic resonance enterography in patients with small bowel Crohn's disease. Gastroenterology. 2018, 154:1172-1194. 10.1053/j.gastro.2017.11.274

9. Zhang W, Zhu W, Li Y, Zuo L, Wang $\mathrm{H}, \mathrm{Li} \mathrm{N}, \mathrm{Li} \mathrm{J}$ : The respective role of medical and surgical therapy for enterovesical fistula in Crohn's disease. J Clin Gastroenterol. 2014, 48:708-711. 10.1097/MCG.0000000000000040

10. Ben-Ami H, Ginesin Y, Behar DM, Fischer D, Edoute Y, Lavy A: Diagnosis and treatment of urinary tract complication in Crohn's disease: an experience over 15 years. Can J Gastroenterol. 2002, 16:225-229.

11. Feagan BG, Sandborn WJ, Gasink C, et al.: Ustekinumab as induction and maintenance therapy for Crohn's disease. N Engl J Med. 2016, 375:1946-1960. 10.1056/NEJMoa1602773 